\title{
Tuberculose vertebral: análise descritiva de uma série de casos submetidos a tratamento cirúrgico
}

\author{
Tuberculosis of the spine: descriptive analysis of a series of cases \\ surgically treated
}

\author{
Tuberculosis vertebral: un análisis descriptivo de una serie de casos \\ sometidos al tratamiento quirúrgico
}

\author{
Luiz Cláudio Schettino' \\ Luís Eduardo Carelli² \\ Marcello Oliveira Barbosa ${ }^{3}$
}

\section{RESUMO}

Objetivo: apresentar a casuística cirúrgica no tratamento da tuberculose vertebral e comparar os resultados com a literatura atual. Métodos: foi realizada a análise retrospectiva dos prontuários dos pacientes submetidos à cirurgia para tratamento da espondilite tuberculosa. Foram estudados o tipo de cirurgia realizada, o estado neurológico e o grau de cifose antes da cirurgia e ao final de 12 meses. O Estado neurológico foi definido pela escala de Frankel modificada. A deformidade cifótica foi medida pelo método de Cobb. Foram identificados 23 pacientes com diagnóstico confirmado de tuberculose vertebral. Foram submetidos a tratamento cirúrgico 13 dos 23 pacientes. Três pacientes foram excluídos e dez pacientes participaram do estudo. Resultados: a maioria dos pacientes foi submetida à descompressão, artrodese anterior e posterior por via combinada ou posterior com instrumentação. Seis dos dez pacientes apresentavam déficit neurológico ao diagnóstico, sendo duas crianças que

\section{ABSTRACT}

Objective: to present our experience on the surgical treatment of spinal tuberculosis and to compare our data to the recent literature. Methods: a retrospective evaluation of patients who underwent surgical procedure to treat the disease was done. We analyzed the type of surgery, neurological status and angle of kyphosis before the surgery and after twelve months. The neurological status was determined by the Frankel scale modified by ASIA. The kyphotic deformity was measured using the Cobb method. We identified 23 patients with confirmed diagnosis of tuberculosis of the spine. Thirteen individuals in this group were operated. Three patients were excluded and ten participated in the study. Results: most of the patients underwent decompression, anterior and/or posterior fusion by combined or posterior approach. Six patients presented neurological deficit at the time of diagnosis. Two of these six were children and presented with Pott's disease on

\section{RESUMEN}

Objetivo: presentar la casuística quirúrgica en el tratamiento de la tuberculosis vertebral y comparar los resultados con la literatura actual. Métodos: se realizó un análisis retrospectivo de las historias clínicas de los pacientes sometidos a la cirugía para el tratamiento de la espondilitis tuberculosa. Fueron estudiados el tipo de cirugía realizada, el estado neurológico y el grado de la cifosis antes de la cirugía $y$ al final de 12 meses. El estado neurológico fue definido por la escala de Frankel modificada. La deformidad cifótica fue medida por el método de Cobb. Fueron identificados 23 pacientes con diagnóstico confirmado de tuberculosis vertebral. Fueron sometidos a tratamiento quirúrgico 13 de los 23 pacientes. Tres pacientes fueron excluidos y diez pacientes participaron del estudio. Resultados: la mayoría de los pacientes fueron sometidos a la descompresión, artrodesis anterior y posterior por vía combinada o posterior con instrumentación. Seis de los diez pacientes presentaban un déficit neurológico al momento del diagnósti-

\footnotetext{
Grupo de Coluna do Instituto Nacional de Traumatologia e Ortopedia - INTO - Rio de Janeiro (RJ), Brasil.

'Chefe do Grupo de Coluna do I Instituto Nacional de Traumatologia e Ortopedia - INTO - Rio de Janeiro (RJ), Brasil.

${ }^{2}$ Mestre em Ortopedia; Médico ortopedista e integrante do Grupo de Coluna do Instituto Nacional de Traumatologia e Ortopedia - INTO - Rio de Janeiro (RJ), Brasil.

${ }^{3}$ Estagiário do Grupo da Coluna do Instituto Nacional de Traumatologia e Ortopedia - INTO - Rio de Janeiro (RJ), Brasil.
} 
apresentavam a lesão denominada de Mal de Pott à admissão. Três pacientes apresentaram recuperação completa da função motora dos membros inferiores. Um paciente apresentou piora do déficit neurológico após a cirurgia devido à resistência bacteriana ao uso de quatro drogas diferentes e à meningite tuberculosa. Em relação às medidas da cifose angular e regional no início e ao final do seguimento, constatou-se que a média da cifose angular no início foi de $26,7^{\circ}\left(0^{\circ}\right.$ a $\left.90^{\circ}\right)$ e de $21,2^{\circ}\left(0^{\circ}\right.$ a $\left.50^{\circ}\right)$ ao final do seguimento. A média da cifose regional no início foi de $24,10^{\circ}\left(-27^{\circ} \mathrm{a}+60^{\circ}\right)$ e de $21^{\circ}\left(-33^{\circ}\right.$ a $\left.+65^{\circ}\right)$ ao final do seguimento. Houve uma redução da média de cifose angular e regional de $5,7^{\circ}$ e $3,1^{\circ}$, respectivamente. Não se observou progressão significativa da cifose em nenhum dos pacientes operados. Conclusão: o tratamento cirúrgico da espondilite tuberculosa nesta série de casos foi eficaz na prevenção da progressão da cifose avaliada em um período mínimo de 12 meses. $\mathrm{O}$ déficit neurológico relacionado à tuberculose vertebral foi mais grave nas crianças.

DESCRITORES: Tuberculose osteoarticular/cirurgia; Manifestações neurológicas; Cifose admission. Three patients presented complete recovery of the motor function of their legs. One patient had neurological status aggravated after the surgery due to bacterial resistance at the administration of four different drugs and to tuberculous meningitis. Regarding to the kyphosis, the mean focal kyphotic angle in the beginning of the follow up was $26,7^{\circ}\left(0^{\circ}\right.$ to $\left.90^{\circ}\right)$ and $21,2^{\circ}\left(0^{\circ}\right.$ to $\left.50^{\circ}\right)$ at the end of the study. The mean regional kyphotic angle was $24,10^{\circ}\left(-27^{\circ}\right.$ to $\left.+60^{\circ}\right)$ in the beginning and $21^{\circ}\left(-33^{\circ}\right.$ to $+65^{\circ}$ ) at the end of the follow-up. There was a decrease of the mean focal and regional kyphosis of 5,7 ${ }^{\circ}$ and $3,1^{\circ}$, respectively. We did not observe important kyphotic angle increase in any operated patient at the end of the study. Conclusion: The surgical treatment was efficient to prevent kyphosis progression. The neurological deficit associated with spinal tuberculosis was critical in children.

KEYWORDS: Tuberculosis, osteoarticular/surgery; Neurologic manifestations; Kyphosis co, siendo que dos niños presentaban la lesión denominada de Mal de Pott en el momento de la admisión. Tres pacientes presentaron recuperación completa de la función motora de los miembros inferiores. Un paciente empeoró su déficit neurológico después de la cirugía debido a la resistencia bacteriana por el uso de cuatro fármacos diferentes y a la meningitis tuberculosa. En relación a las medidas de la cifosis angular y regional en el inicio y al final del seguimiento, se constató que el promedio de la cifosis angular al inicio fue de $26.7^{\circ}$ $\left(0^{\circ}\right.$ a $\left.90^{\circ}\right)$ y de $21.2^{\circ}\left(0^{\circ}\right.$ a $\left.50^{\circ}\right)$, al final del tratamiento. El promedio de la cifosis regional al inicio fue de $24.10^{\circ}\left(-27^{\circ}\right.$ $\left.a+60^{\circ}\right)$ y de $21^{\circ}\left(-33^{\circ} a+65^{\circ}\right)$ al final del seguimiento. Hubo una reducción del promedio de la cifosis angular y regional de $5.7^{\circ}$ y $3.1^{\circ}$, respectivamente. No fue observada progresión significativa de la cifosis en ninguno de los pacientes operados. Conclusión: el tratamiento quirúrgico de la espondilitis tuberculosa en esta serie de casos fue eficaz en la prevención de la progresión de la cifosis evaluada en un periodo mínimo de 12 meses. El déficit neurológico relacionado a la tuberculosis vertebral fue más grave en los niños.

\section{DESCRIPTORES: Tuberculosis} osteoarticular/cirugía; Manifestaciones neurológicas; Cifosis

\section{INTRODUÇÃO}

A tuberculose vertebral é uma doença grave que pode levar à destruição dos corpos vertebrais e deformidades acentuadas da coluna dorsal. Pode estar associada à doença pulmonar ou não. Segundo a Organização Mundial de Saúde (OMS), a cada ano surgem 8 milhões de novos casos de tuberculose, com cerca de 20 milhões de casos de infecção no mundo, sendo 1,7 bilhões de infectados no terceiro mundo ${ }^{1}$. A tuberculose mata cerca de 3 milhões de pessoas por ano em todo o mundo ${ }^{1,2}$. Cerca de $10 \%$ dos casos clínicos de tuberculose acometem o osso ${ }^{2}$. A tuberculose vertebral é a mais frequente e representa cerca de metade dos casos de tuberculose óssea ${ }^{1-3}$. Dentre as deformidades encontradas na coluna, destacam-se a escoliose e a cifose. A cifotização ou cifose progressiva ocorre com mais frequência nos casos em que a área envolvida compreende a coluna torácica baixa e a transição toracolombar. A espondilodiscite tuberculosa pode acometer um ou mais segmentos da coluna vertebral (Figuras 1 e 2). O envolvimento da coluna cervical é raro, relaciona-se a uma alta taxa de mortalidade enquanto o envolvimento de três ou mais vértebras traduz um risco elevado de paraplegia ${ }^{4,5}$. O tratamento desta patologia permanece controverso. A drenagem de abscessos e a curetagem dos fragmentos ósseos e de disco sequestrados seguida da colocação de órteses podem levar ao agravamento da cifose ${ }^{6}$. O tratamento quimioterápico isolado está relacionado a uma alta incidência de progressão da cifose e a uma baixa taxa de fusão dos corpos vertebrais $^{6,7}$ (Figuras 3 e 4). A indicação cirúrgica de rotina nos casos de tuberculose vertebral não é aceita. A pré-determinação de quais pacientes estariam mais suscetíveis a uma cifose grave ou cifotização progressiva poderia guiar a tomada de decisão clínica. Rajasekaran et al. descreveram um método preditivo, o viés foi de que 




Figuras 1 e 2

Paciente de 54 anos do sexo feminino. Tomografia computadorizada da coluna toracolombar mostra envolvimento multifocal onde se nota a destruição do corpo vertebral com extensão para o pedículo esquerdo em T12 e lesão osteolítica no corpo vertebral de S1.

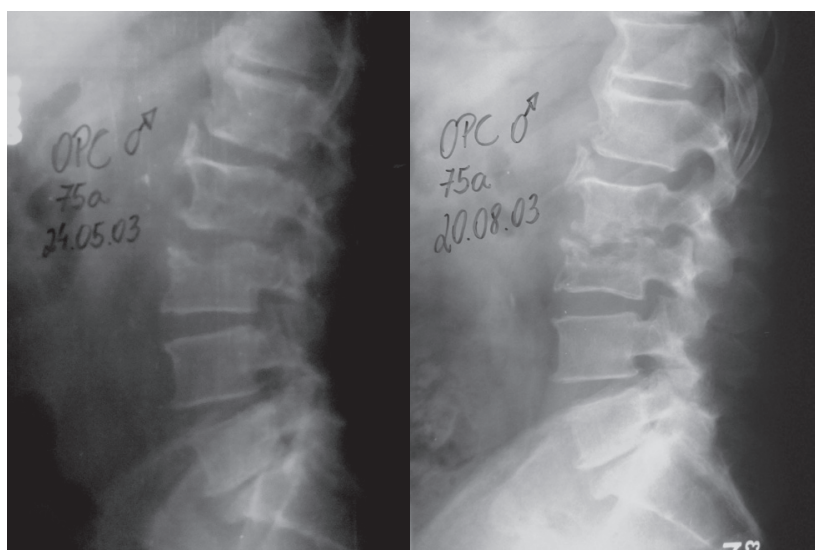

Figuras 3 e 4

Paciente com 75 anos do sexo masculino. Radiografias em perfil da coluna lombar mostrando da figura 3 para a figura 4 a evolução da doença com destruição focal dos corpos vertebrais de $\mathrm{L} 2$ e $\mathrm{L} 3$ e do disco intervertebral correspondente com retificação da lordose em menos de 3 meses de seguimento.

incluíram pacientes com cifose severa que já tinham uma indicação cirúrgica evidente. A acurácia publicada deste método apresentou uma ampla variação, de 34 a $90 \% \%^{5,8}$. A abordagem cirúrgica com drenagem de abscessos e artrodese pela via anterior ou associada à via posterior demonstrou bons resultados quando comparada à quimioterapia isolada, em especial nas crianças com déficit neurológico progressivo e paraplegia já instaladas. Mayer e Adams compararam o tratamento conservador com a artrodese posterior em crianças, concluindo que nenhum tratamento foi superior ao outro na fase aguda da doença ${ }^{3}$. No estágio tardio, no entanto, havendo três ou mais vértebras colapsadas, a artrodese mostrou-se superior, associada a uma menor progressão da cifose ${ }^{3}$.

\section{OBJETIVO}

Relatar a experiência no tratamento cirúrgico da espondilite tuberculosa, comparando os resultados com a literatura.

\section{MÉTODOS}

Análise retrospectiva de 67 prontuários de pacientes do Instituto Nacional de Traumatologia e Ortopedia (INTO) com diagnóstico de espondilodiscite no período de janeiro de 1998 a dezembro de 2007. Destes, 23 pacientes apresentaram comprovação clínica ou anatomopatológica para tuberculose vertebral. Treze deles foram submetidos à cirurgia. Foram excluídos do trabalho os pacientes que tiveram menos de 12 meses de acompanhamento, prontuário incompleto e avaliação radiográfica inadequada. Dez pacientes foram incluídos no estudo. Utilizou-se o método de Cobb para análise radiográfica das deformidades. Foi realizada a medida da cifose angular através das placas terminais preservadas imediatamente caudais e craniais ao nível acometido e a cifose regional, utilizando-se os platôs vertebrais superior de T2 e inferior de T10 para deformidades torácicas, superior de T11 e inferior de L2 para deformidades toracolombares e superior de L2 e inferior de L5 para deformidades lombares ao diagnóstico e ao final do seguimento. $\mathrm{O}$ estado neurológico dos pacientes teve como critério de avaliação a escala de Frankel modificada pela ASIA (American Spine Injury Association). Os pacientes foram analisados quanto à idade, sexo, nível vertebral acometido, quadro clínico, deformidade da coluna ao diagnóstico, tipo de tratamento e deformidade ao final do seguimento de no mínimo um ano.

\section{RESULTADOS}

A distribuição por sexo foi oito mulheres e dois homens. A média de idade foi de 49,5 anos variando de 1 ano e 7 meses a 82 anos. Todos os pacientes queixavam-se de dor. Além do quadro álgico, quatro pacientes apresentaram fraqueza de membros inferiores; dois queixaram-se de emagrecimento; e um paciente queixou-se de febre. Um dos pacientes que se queixou de emagrecimento, o número 6 (Tabela 1), apresentou tuberculose miliar associada e alegou perda de $30 \mathrm{~kg}$ em um período de 6 meses. Um paciente queixou-se de deformidade. O nível vertebral mais acometido foi a transição toracolombar em 4 pacientes (40\%), seguida do nível torácico em outros 3 pacientes. A vértebra mais acometida foi T12. No nível torácico, a vértebra mais acometida foi $\mathrm{T} 5$ e, no nível lombar, foi L2. $\mathrm{O}$ nível cervical não foi acometido na nossa casuística. $\mathrm{O}$ tempo médio de evolução da doença foi de 31 meses, variando de 2 a 180 meses. O tempo médio de segmento foi de 40,4 meses, com mínimo de 12 e máximo de 111 meses.

$\mathrm{O}$ esquema quimioterápico foi realizado em todos os pacientes e variou com o uso de duas, três e quatro drogas. Os antimicrobianos usados foram: rifampicina, isoniazida, pirazinamida e etambutol. O tempo de uso também variou de 6 a 12 meses. O esquema RIP (Rifampicina, Isoniazida e Pirazinamida) foi o mais usado, em 7 pacientes e por período de 6 meses, administrando-se a rifampicina $600 \mathrm{mg} /$ dia, a isoniazida $300 \mathrm{mg} /$ dia e a pirazinamida $2,0 \mathrm{~g} /$ dia nos primeiros dois meses seguidos de isoniazida e rifampicina por mais 4 meses, diariamente, nas mesmas dosagens. 
TABELA 1 - Distribuição dos pacientes por prontuário, idade, sexo, nível acometido e tipo de cirurgia realizada

\begin{tabular}{|c|c|c|c|c|c|c|}
\hline Número & Prontuário & Idade & Sexo & Vértebras & Nível & Conduta \\
\hline 1 & 138515 & 63 & $\mathrm{~F}$ & $\begin{array}{l}\mathrm{T} 11 \text { e T12 } \\
\mathrm{T} 11 \mathrm{~T} 12\end{array}$ & Toracolombar & Drenagem de abscesso \\
\hline 2 & 145286 & 54 & $\mathrm{~F}$ & e L5 & Toracolombar & Descompressão e artrodese via combinada \\
\hline 3 & 107593 & 24 & $\mathrm{~F}$ & L1 e L2 & Lombar & Descompressão e artrodese $360^{\circ}$ via posterior \\
\hline 4 & 210650 & $1 \mathrm{a} 7 \mathrm{~m}$ & $\mathrm{~F}$ & $\begin{array}{l}\text { T3,T4 e } \\
\text { T5 }\end{array}$ & Torácico & $\begin{array}{c}\text { Descompressão e artrodese via posterior com } \\
\text { costotransversectomia }\end{array}$ \\
\hline 5 & 174060 & 73 & $\mathrm{~F}$ & T7 e T8 & Torácico & $\begin{array}{c}\text { Descompressão e artrodese } 360^{\circ} \text { via posterior } \\
\text { usando instrumental de HARTSHILL }\end{array}$ \\
\hline 6 & 139461 & 80 & M & L2 e L3 & Lombar & Descompressão e artrodese via combinada \\
\hline 7 & 156779 & 82 & $\mathrm{~F}$ & $\mathrm{~T} 11$ e T12 & Toracolombar & $\begin{array}{l}\text { Descompressão e artrodese } 360^{\circ} \text { via posterior } \\
\text { com costotransversectomia }\end{array}$ \\
\hline 8 & 145581 & 9 & $\mathrm{~F}$ & $\begin{array}{l}\text { T5, T6 e } \\
\text { T7 }\end{array}$ & Torácico & $\begin{array}{c}\text { Descompressão e artrodese } 360^{\circ} \text { via posterior } \\
\text { com costotransversectomia }\end{array}$ \\
\hline 9 & 104230 & 59 & $\mathrm{~F}$ & $\mathrm{~L} 2, \mathrm{~L} 3$ e $\mathrm{L} 4$ & Lombar & $\begin{array}{l}\text { Descompressão e artrodese via combinada } \\
\text { com enxerto de fíbula anterior }\end{array}$ \\
\hline 10 & 156522 & 50 & M & T12 e L1 & Toracolombar & Descompressão e artrodese via combinada \\
\hline
\end{tabular}

Foram submetidos à artrodese circunferencial de $360^{\circ} 9$ pacientes $(90 \%)$. Foi utilizada a via combinada em quatro pacientes e a via posterior em cinco pacientes (Tabela 1). Em cinco pacientes, foi utilizada a cesta de Harms na artrodese anterior (Figuras 5 e 6). Um paciente utilizou enxerto de fíbula. Foi indicada a artrodese anterior com colocação de cesta na paciente 3 , porém não foi realizado devido a aderências na loja renal esquerda, o que impediu o deslocamento daquele rim. A paciente apresentava rim único. Foi submetida à nefrectomia à direita por tuberculose renal prévia e apresentava fístula ativa de baixo débito na cicatriz cirúrgica no momento da internação. Foi

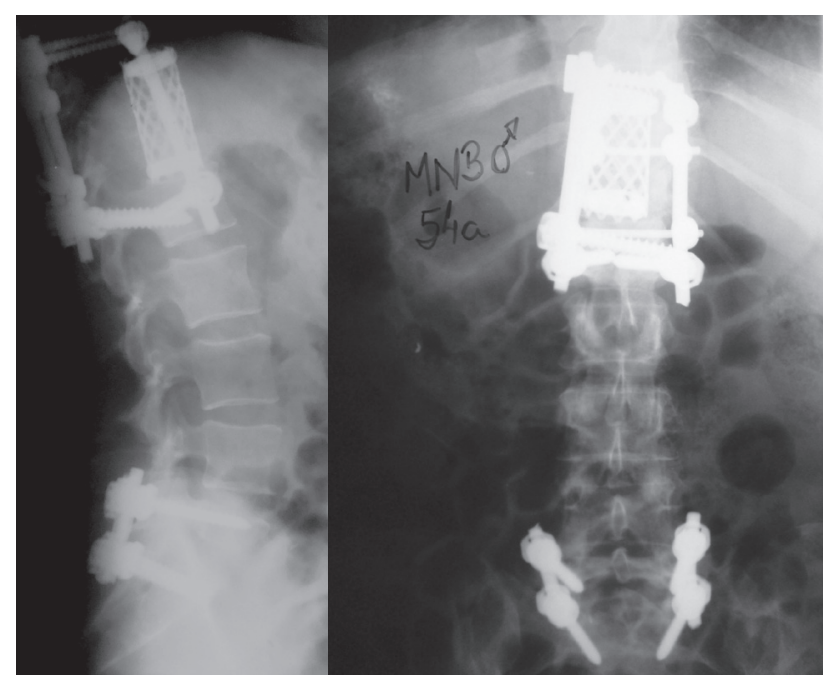

Figuras 5 e 6

Paciente de 54 anos do sexo feminino. Radiografias pósoperatórias de perfil e em AP da coluna toracolombar. Notar a artrodese anterior com enxerto usando a cesta de Harms e a instrumentação anterior e posterior no segmento T1 1 - L T. O acometimento de L5-S1 foi tratado com descompressão, enxertia via anterior e instrumentação posterior. então realizada apenas a drenagem de abscesso do psoas esquerdo pela via anterior. O paciente 4 foi submetido à artrodese por via posterior com costotransversectomia para drenagem de abscesso e colocação de enxerto de ilíaco. A paciente 5 foi submetido à artrodese anterior em outro serviço e foi reoperada devido a pseudoartrose e colapso de T7, constatados pela tomografia computadorizada.

Quanto às complicações, o paciente 9 apresentou falência do material de síntese caracterizada por afrouxamento dos parafusos pediculares após 14 meses de cirurgia. Foi realizada a retirada do material de síntese e constou-se a artrodese vertebral durante o ato operatório. Não foram observadas outras complicações no período de seguimento dos pacientes.

O estado neurológico dos pacientes antes e ao final do seguimento é mostrado na tabela 2. Seis pacientes apresentavam algum déficit neurológico no pré-operatório. Destes, três apresentaram melhora do déficit neurológico ao final do seguimento. Os outros quatro pacientes mantiveram o estado neurológico normal inalterado após o final do seguimento. Destes, um paciente apresentava apenas

TABELA 2 - Estado neurológico no préoperatório e ao final do seguimento

\begin{tabular}{lcc}
\hline Número & Pré-operatório & Final \\
\hline 1 & E & E \\
2 & E & E \\
3 & E & E \\
4 & C & E \\
5 & B & B \\
6 & D & E \\
7 & D & D \\
8 & D & B \\
9 & C & E \\
10 & E & E \\
\hline
\end{tabular}


bexiga neurogênica no pré-operatório. Dois pacientes não apresentaram melhora ao final do seguimento. Um paciente apresentou meningite tuberculosa durante a internação hospitalar e piora do estado neurológico após a cirurgia, evoluindo do ASIA "D" para o "B".

Quanto ao grau de cifose, observou-se que, considerando as medidas da cifose angular e regional no início e ao final do seguimento, a média da cifose angular no início foi de $26,7^{\circ}\left(0^{\circ}\right.$ a $\left.90^{\circ}\right)$ e de $21,2^{\circ}\left(0^{\circ}\right.$ a $\left.50^{\circ}\right)$ ao final do seguimento (Tabela 3 ). A média da cifose regional no início foi de $24,10^{\circ}\left(-27^{\circ}\right.$ a $\left.+60^{\circ}\right)$ e de $21^{\circ}\left(-33^{\circ}\right.$ a $\left.+65^{\circ}\right)$ ao final do seguimento. Houve uma redução da média de cifose angular e regional de $5,7^{\circ}$ e $3,1^{\circ}$, respectivamente. Ao analisar cada segmento torácico, toracolombar e lombar separadamente, verificou-se que a média da cifose angular no início na coluna torácica foi de $51,66^{\circ}\left(15^{\circ}\right.$ a $\left.90^{\circ}\right)$ e da cifose regional foi de $60^{\circ}\left(35^{\circ}\right.$ a $\left.84^{\circ}\right)$. Ao final do segmento, a média da cifose angular na coluna torácica foi de $36,66^{\circ}$ $\left(14^{\circ}\right.$ a $\left.50^{\circ}\right)$ e a média da cifose regional foi de $50^{\circ}\left(40^{\circ}\right.$ a $\left.65^{\circ}\right)$. Em relação à coluna toracolombar, a média da cifose angular no início foi de $23^{\circ}$ e a da cifose regional foi de $23,75^{\circ}$. No final do seguimento, as médias de cifose angular e regional foram $21,5^{\circ}$ e $25,5^{\circ}$. Analisando-se a coluna lombar, foi constatado que a média da cifose angular no início foi de $6,66^{\circ}\left(0^{\circ}\right.$ a $\left.16^{\circ}\right)$ e da regional foi $-11^{\circ}\left(-27^{\circ}\right.$ a $\left.0^{\circ}\right)$. No final, a média da cifose angular na coluna lombar foi de $5,3^{\circ}$ e da cifose regional foi de $-14^{\circ}$.

\section{TABELA 3 - Curvas de cifose no início e ao final do seguimento}

\begin{tabular}{lcccc}
\hline Número & \multicolumn{2}{c}{$\begin{array}{c}\text { Cifose focal } \\
\text { (em graus) }\end{array}$} & \multicolumn{2}{c}{$\begin{array}{c}\text { Cifose regional } \\
\text { (em graus) }\end{array}$} \\
\hline & Início & Final & Início & Final \\
1 & 26 & 28 & 16 & 21 \\
2 & 21 & 8 & 10 & 1 \\
3 & 0 & 0 & 0 & 0 \\
4 & 50 & 46 & 61 & 65 \\
5 & 15 & 14 & 35 & 40 \\
6 & 4 & 0 & -27 & -33 \\
7 & 22 & 25 & 55 & 60 \\
8 & 90 & 50 & 84 & 45 \\
9 & 16 & 16 & -7 & -9 \\
10 & 23 & 25 & 14 & 20 \\
\hline
\end{tabular}

\section{DISCUSSÃO}

Mais de 2 milhões de pessoas sofrem no mundo com a tuberculose ativa na forma espinhal. Observamos, em nosso estudo, que a dor é o sintoma que predomina na coluna. Esse dado é corroborado pela literatura ${ }^{3}$. O diagnóstico da tuberculose pode ser realizado por métodos clínicos, radiológicos e laboratoriais ou acrescidos da biópsia. Todos os pacientes operados foram submetidos à biópsia transpedicular do local da lesão. O exame anatomopatológico mostrou-se inconclusivo em dois pacientes e sugestivo de tuberculose em oito pacientes pela presença de infil- trado inflamatório de mononucleares, necrose e formação granulomas. Não foi evidenciada a presença do bacilo de Koch em nenhuma das amostras: a coloração de BAAR foi negativa.

O déficit neurológico afetou seis dos dez pacientes estudados. As duas crianças incluídas no estudo apresentavam déficit neurológico na internação. Ambas apresentaram envolvimento torácico, colapso de três vértebras adjacentes e o abscesso paravertebral caracterizando o mal de Pott (Figura 7). Uma das crianças apresentou meningite tuberculosa e piora do estado neurológico, evoluindo para paraplegia ao final do segmento. Bailey et al. encontraram evidência neurológica de mielopatia na admissão de 43 dentre 100 crianças que foram submetidas ao tratamento pelo método de Hong Kong (Desbridamento, ressecção radical anterior e artrodese). Destas, 20 apresentavam-se paraplégicas ${ }^{6}$. Rajasekaran et al. encontraram uma média de envolvimento de 3,1 corpos vertebrais em 12 crianças com idade abaixo de 10 anos e uma média de perda (destruição) dos corpos vertebrais de 2,2, comparado a média de 1,9 vértebras envolvidas e de 0,87 de perda vertebral em 53 pacientes participantes do estudo Madras de espondilite tuberculosa, o que foi estatisticamente significativo

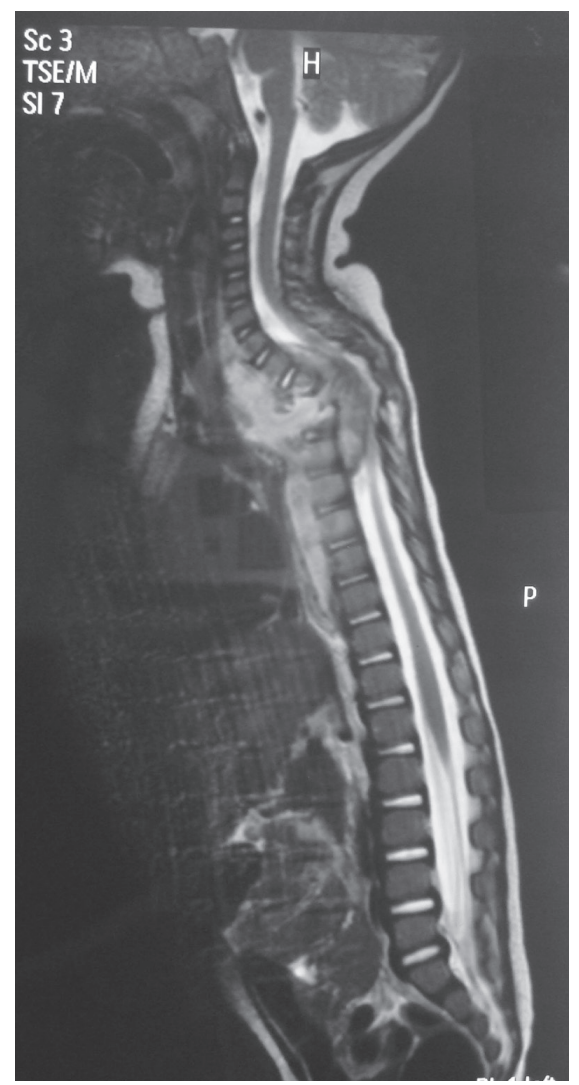

Figura 7

Imagem de ressonância magnética de paciente de 1 ano e 7 meses do sexo feminino. Corte sagital mostrando destruição dos corpos vertebrais de T3, T4 e T5, cifose acentuada da coluna torácica e grande abscesso epidural comprimindo a medula espinhal. 
$(\mathrm{p}<0,01)^{8}$. Jain e Dhammi afirmaram que, nas crianças, a destruição vertebral é mais severa porque a maior parte do osso é cartilaginosa ${ }^{7,9}$. A medula espinhal pode ser comprimida pelo pus, tecido de granulação, caseum, disco ou sequestro ósseo. O prontuário da criança relata resistência do bacilo de Koch à associação de quatro drogas diferentes e infecção de ferida pós-operatória. Achamos que a meningite tuberculosa e a piora do déficit motor estejam relacionadas à invasão e destruição do tecido neural da medula por estas cepas resistentes da micobacteria. Por outro lado, três dos seis pacientes que apresentavam déficit motor tiveram recuperação total ao final do seguimento, o que demonstra um grande benefício do tratamento cirúrgico nestes pacientes (Figura 8). Não houve influência danosa da intervenção cirúrgica no estado neurológico dos pacien-

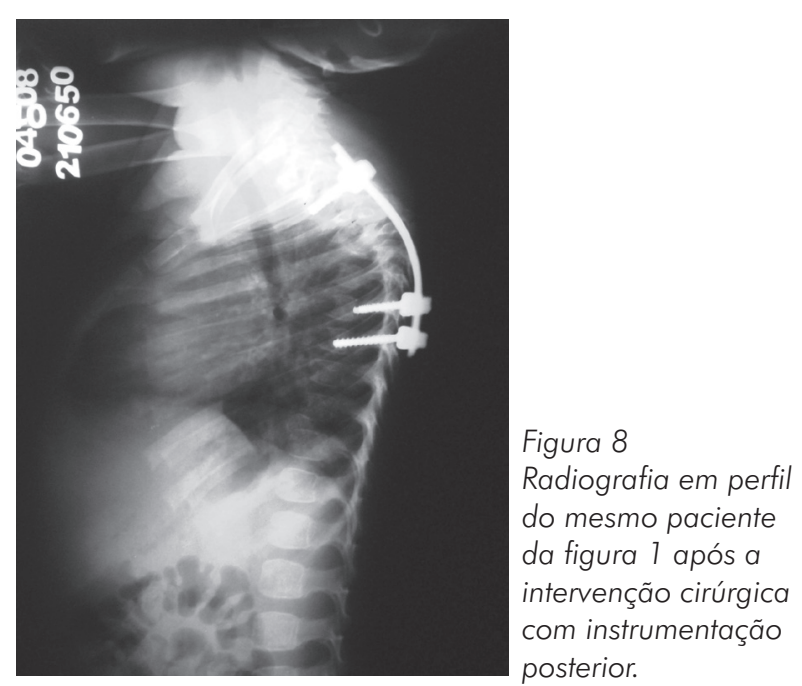

tes, pois todos que se apresentavam sem déficit antes da cirurgia permaneceram assim ao final do seguimento.

A descompressão cirúrgica precoce deve ser realizada nos casos de surgimento de déficit neurológico ou quando este permanece inalterado ou apresenta piora após o início do tratamento clínico (repouso no leito e drogas antituberculose). Incluem-se nesses casos a paraplegia de início súbito com complicações neurológicas severas desenvolvidas em um curto período de tempo e a paraplegia com envolvimento do arco neural ${ }^{8}$. A cirurgia é realizada para descomprimir a medula espinhal e preencher o defeito resultante com o objetivo de alcançar a cicatrização da lesão. Em lesões anteriores da vértebra, a abordagem deve ser anterior. Acreditamos que uma laminectomia em uma lesão vertebral anterior seja desastrosa, pois remove o único segmento sadio da coluna vertebral, tornando-a instável ${ }^{9,10}$. Com relação ao enxerto utilizado na artrodese anterior, existem várias técnicas descritas na literatura que empregam enxerto autólogo da crista ilíaca, costela, fíbula, aloenxerto de fêmur e úmero ou colocação de enxerto esponjoso impactado em cestas de titânio. $\mathrm{O}$ uso do enxerto autólogo tem como principais vantagens a consolidação em tempo menor, a sua ação osteoindutora e a imunocompatibilidade. As desvantagens são a disponibilidade óssea limitada, o suporte estrutural inadequado, que permite a progressão da cifose e a morbidade do sítio doador ${ }^{9}$. Adotamos a cesta de Harms na artrodese anterior devido ao melhor suporte vertebral, diminuindo assim o risco de progressão da cifose após a cirurgia. Quanto à instrumentação, sabemos que a instabilidade vertebral tende a aumentar no pós-operatório imediato após a descompressão e enxertia. $\mathrm{O}$ enxerto ósseo não promove estabilidade quando inserido 9 . A grande maioria dos estudos científicos da última década publicaram o uso de instrumentação anterior ou posterior visando à correção da cifose ou a prevenção da progressão pós-operatória dessa deformidade. Além disso, o seu uso permite uma maior mobilidade pósoperatória até a incorporação do enxerto, evitando que o paciente permaneça confinado ao leito. Os pacientes nos quais não é realizada a instrumentação estão mais suscetíveis a complicações como a fratura ou deslocamento do enxerto ósseo. A instrumentação posterior com descompressão e artrodese anterior podem ser realizadas em um ou dois tempos. Quando é realizada em um tempo operatório, apresenta grande morbidade. Se realizada em dois tempos, a correção da cifose não é alcançada efetivamente ${ }^{9,11}$. A quimioterapia deve ser associada ao tratamento operatório e iniciada após a confirmação diagnóstica. Parthasarathy et al. recomendam a associação do esquema RIP com etambutol $(30 \mathrm{mg} / \mathrm{kg} / \mathrm{dia})$ nos primeiros dois meses seguida de isoniazida e rifampicina por mais quatro meses, todas as drogas administradas três vezes por semana no tratamento da tuberculose ativa da coluna torácica ou lombar sem paraplegia ${ }^{12}$.

Neste estudo, não houve uma progressão significativa da cifose ao final do seguimento de acordo com as médias de cifose focal e regional encontradas, utilizando-se o enxerto ósseo com cesta anterior e a instrumentação posterior. A cifotização pode ocorrer durante os 18 meses subsequentes à cirurgia ${ }^{12}$. Sendo assim, podemos afirmar que esse método de tratamento, empregado na maioria dos pacientes do estudo, foi eficaz em evitar a progressão da cifose. Notou-se ainda que, ao separar os pacientes por segmento vertebral acometido, a deformidade cifótica era mais acentuada nos pacientes com envolvimento torácico e praticamente ausente nos três pacientes com envolvimento lombar que apresentavam apenas retificação da lordose. Tal fato é corroborado pela literatura, que mostra que os pacientes cujos segmentos torácico e toracolombar são acometidos apresentam mais cifose quando comparados às espondilites tuberculosas do segmento lombar ${ }^{8,11,13,14}$.

\section{CONCLUSÃO}

Nesta série de casos confirmados de espondilodiscite tuberculosa, o tratamento cirúrgico foi eficaz na prevenção da progressão da cifose em um período de seguimento mínimo de 12 meses. O déficit neurológico relacionado à tuberculose vertebral foi mais grave nas crianças. A instrumentação cirúrgica foi importante, pois preveniu a progressão da deformidade cifótica. 


\section{REFERÊNCIAS}

1. Sundararaj GD, Behera S, Ravi V, Venkatesh K, Cherian VM, Lee V. Role of posterior stabilisation in the management of tuberculosis of the dorsal and lumbar spine. J Bone Joint Surg Br. 2003;85(1):100-6.

2. Wen-Jer $\mathrm{C}$, Wu CC, Jung $\mathrm{CH}$, Chen LH, Niu CC, Lai PL. Combined anterior and posterior surgeries in the treatment of spinal tuberculous spondylitis. Clin Orthop Relat Res. 2002;(398):50-9.

3. Jain AK, Aditya VM, Santosh J. Kyphus correction in spinal tuberculosis. Clin Orthop Relat Res. 2007(460):117-23.

4. Konstam P. Is your operation necessary? In: Leach RE, Hoaglund FT, Riseborough E, editors. Controversies in orthopaedic surgery Philadelphia: WB. Saunders; 1982. p. 487-503.

5. Rajasekaran S, Shanmugasundaram TK. Prediction of the Angle of Gibbus deformity in tuberculosis of the spine. J Bone and Joint Surg Am. 1987;69(4):503-9.
6. Bailey HL, Gabriel M, Hodgson AR, Shin JS. Tuberculosis of the spine in children. Operative findings and results in one hundred consecutive patients treated by removal of the lesion and anterior grafting. J Bone and Joint Surg Am. 1972;54(8): 1633-57.

7. Jain AK. Treatment of tuberculosis of the spine with neurologic complications. Clin Orthop Relat Res. 2002(398):75-84.

8. Rajasekaran S, Shanmugasundaram TK, Prabhakar R, Dheenadhayalan J, Shetty AP, Shetty DK. Tuberculous lesions of the lumbosacral region: a 15-year follow-up of patients treated by ambulant chemotherapy. Spine. 1998;23(10):1163-7.

9. Jain AK, Dhammi IK. Tuberculosis of the spine: a review. Clin Orthop Relat Res. 2007(460):39-49.

10.Souza PS, Puertas EB, Wajchenberg M, Oliveira VM, Oliveira CE, D’Orto CC. Tuberculose óssea na coluna vertebral: aspectos clínicos e cirúrgicos. Tuberculose óssea na coluna vertebral: aspectos clínicos e cirúrgicos. Coluna/Columna. 2005;4(2):75-80.
11. Shankar A, Gurdeep SR. Posterior spinal cord compression: outcome and results. Spine. 2006;31(17):E574-8.

12.Partasarathy R, Sriram K, Santha T, Prabhakar R, Somasundaram PR, Sivasubramanian S. Short-course chemotherapy for tuberculosis of the spine: a comparison between ambulant treatment and radical surgery- ten year report. J Bone Joint Surg Br. 1999;81(3):464-71.

13.Daher S, Cardoso ALP, Souza Junior ZA, Pimenta Junior WE, Moraes FB, Daher MT, et al. Tuberculose espinhal: avaliação de 26 casos. Coluna. 2006;5(1):1-6.

14.Narazaki DK, Correia LS, Araújo MP, Cristante AF, Iutaka AS, Marcon RM, et al. Deformidade residual na tuberculose da coluna vertebral. Coluna. 2008;7(1):23-6.

\section{Correspondência}

Marcello Oliveira Barbosa

Rua 36 Sul Lote 13, Residencial Villa Pavanelli II, Apto 503 - Águas Claras

CEP 71931-360 - Brasília (DF), Brasil

E-mail: mobarbos@terra.com.br 